\title{
Consultation for physical illnesses by patients diagnosed and treated for psychiatric disorders by a general practitioner: 20 year follow up study
}

\author{
Greg Wilkinson, Nigel Smeeton, David Skuse, John Fry
}

\begin{abstract}
Patterns of consultation for physical illness were analysed in a body of consultation data covering a continuous 20 year period from a single general practitioner in south London. Three groups of adult patients were identified: patients with a psychiatric disorder and a new prescription for a psychotropic drug; patients with a psychiatric disorder but no new prescription for a psychotropic drug; and a control group without psychiatric disorder. The percentages of patients having one or more consultation for physical illnesses were stable over the years studied, being roughly $90 \%, 85 \%$, and $60 \%$ respectively. For groups identified in 1972 their patterns of consultation for physical illness were examined in 1957,1962 , and annually from 1967 to 1976 . In every year studied except 1957 the group with a psychiatric disorder and a new prescription for a psychotropic drug exceeded both other groups in the percentage of patients having one or more consultations for physical illnesses. Both groups with psychiatric disorders had an excess of consultations over the control group; this excess halved in about four years for the group with a new prescription and in about one year for the group without a new prescription.

Mental health care by general practitioners for patients with psychiatric disorders does not seem to have an offset effect on general health care of these patients.
\end{abstract}

\section{Introduction}

In 1967 a reduction in the use of medical care facilities was reported among people enrolled with Kaiser Permanente health maintenance organisation who had received mental health services. ${ }^{1}$ Since then several studies from the United States have investigated the "offset effect": whether the provision of mental health care reduces the demand for general medical services. ${ }^{2.5}$ Analysis of 58 controlled studies together with a search of the claims files for the Blue Cross and Blue Shield federal employees' plan for 1974-8 suggested that providing outpatient mental health services did seem to reduce the demand for general medical services. ${ }^{6}$ The overall conclusion is that in the short term patients receiving specialist mental health care use general medical services less frequently than do untreated patients. An important proviso may be added: it has recently become apparent that, although mental health care may be associated with offset savings in use of medical care and related charges, the visits and charges for specialist treatment may boost total use and charges (non-psychiatric plus specialist mental health) above those of patients treated solely by non-psychiatric physicians.

In the light of these reports, we decided to look for an offset effect in the context of general practice in
Britain: firstly, because there do not seem to be any investigations in the United Kingdom of this topic, which helps to illuminate the associations between physical and mental disorders, patterns of use of health care, and the impact of health services; and, secondly, because most patients with mental disorders are treated in general practice. ${ }^{89}$ For this purpose we obtained consultation data for a continuous 20 year period from a single general practitioner in south London.

\section{Materials and methods}

In 1957 the general practitioner began compiling a case register of all 6346 patients then registered in his practice; 1530 of them were still registered in 1976. There were no major changes in his practice during the period. The general practitioner's detailed NHS records provided both a prospective design and a satisfactory level of reliability. The patients who were studied were limited to those who had remained registered with the general practitioner; moreover, the general practitioner's diagnostic habits were known and his assessment of neurotic and chronic psychiatric disorder was comparable to that of a psychiatrist. ${ }^{10} 11$ The rate of detection of psychiatric cases was similar to that in a large random sample of British general practitioners. ${ }^{12}$

The records for the 726 men and 804 women registered with the practice from 1957 to 1976 had been coded for number of visits per year, psychiatric disorders (depressive neurosis, anxiety and phobic neuroses, personality disorder, functional psychosis, drug-induced psychoses and drug reactions, dementia and organic mental disorders, miscellaneous neurotic disorders, and alcohol abuse and drug dependence), physical disorders (locomotor, gastrointestinal, cardiovascular, neurological, upper and lower respiratory tract disorders, gynaecological disorders and pregnancy, sexual problems and genitourinary disorders, new growths, hypertension, metabolic and endocrine disorders, skin conditions, accidents and trauma, infectious diseases, and "other" physical disorders), and new prescriptions for psychotropic drugs (antidepressants, minor and major tranquillisers, barbiturates prescribed for daytime sedation, and amphetamines unless prescribed for slimming; the drug had not been prescribed in the past six months).

We restricted our analysis to patients aged 25 years and over in selected years $(1957,1967,1972$, and 1976). Three groups were identified: patients diagnosed by the general practitioner as having a psychiatric disorder and receiving a new prescription for a psychotropic drug within the year; patients diagnosed by the general practitioner as having a psychiatric disorder but not receiving a new prescription for a psychotropic drug within the year; and 
a control group of patients with no diagnosis of psychiatric disorder within the year. Patterns of consultation for physical illnesses are presented as the percentage of patients in' each group who had one or more consultations for physical illnesses within the year being considered. The data were standardised for sex.

Firstly, to determine whether patterns of consultation for physical illnesses differed among the three groups over the two decades, we examined the patterns in 1957, 1967, 1972, and 1976. Secondly, to seek an offset effect we took the three groups identified in the index year 1972 and looked at their patterns of consultation for physical illnesses in 1957, 1962, and each year from 1967 to 1976 . As data will later become available to extend these observations to 30 years 1972 , the midpoint of the period 1957-87, was used as the index year.

\section{Results}

The mean rate for consultations in all patients each year was four for women and two for men. All patients attended at least once in the two decades and only $12.5 \%$ women and $25 \%$ men attended in 10 or fewer years. Overall, $75 \%$ of the women and $50 \%$ of the men were seen at least once for a problem regarded by their doctor as largely or wholly psychiatric in nature..$^{13}$

The percentages of patients having one or more consultations for physical illness were similar within each study group in 1957, 1967, 1972, and 1976, being about $90 \%$ for patients with psychiatric illness with a new prescription for psychotropic drugs, $85 \%$ for patients with psychiatric illness and no new prescription, and $60 \%$ for patients without psychiatric illness. In each year a preponderance $(5: 1)$ of women having psychiatric illness but no new prescription consulted their doctor for physical illness. This was a significant difference compared to other groups, which had roughly equal numbers of women and men consulting the doctor $\left(p \leqslant 0.001, \chi^{2}\right.$ test). There were no significant differences in consultation rates for
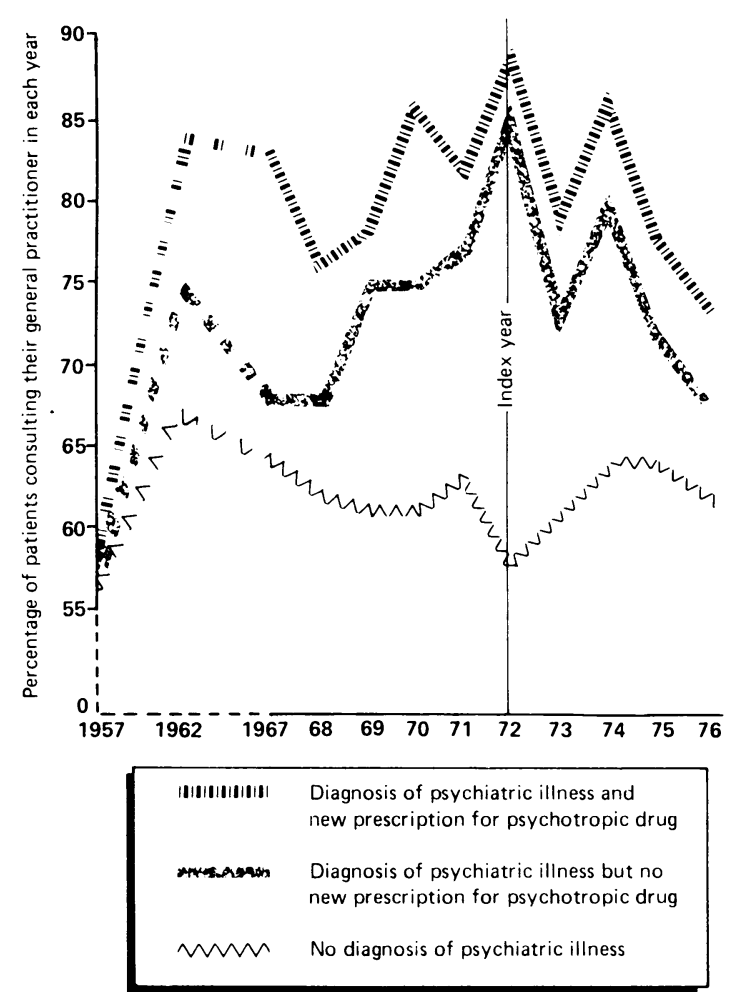

Patients with and without a diagnosis of psychiatric illness who consulted their general practitioner for symptoms of physical illness at least once in each year. Data are for 599 men and 705 women and are standardised for sex physical illness among age groups (25-44, 45-65, over 65 years) in each study group.

To assess any offset effect we examined the patterns of consultation for physical illness in 1957, 1962, and 1967-76 for the study groups identified in the index year 1972. Of the 1530 patients registered with the general practitioner throughout the period, 1304 (599 men and 705 women) were aged over 25 and consulted the doctor in the index year. Of these, 96 $(7 \cdot 8 \% ; 43$ men and 53 women) had psychiatric illness and a new prescription for psychotropic drugs, $143(11 \cdot 7 \% ; 24$ men and 119 women) had psychiatric illness but no new prescription, and $1065(90.5 \%$; 532 men and 533 women) were in the control group. Most patients $(1227 ; 94 \%)$ were aged over 18 throughout the period.

The percentages of patients in each group consulting the doctor for physical illness in 1957, 1962, and 1967-76 are shown in the figure. No offset effect was found; on the contrary, in every year except 1957 a greater percentage of patients in the group with psychiatric illness and a new prescription for psychotropic drugs than in other groups consulted the doctor. The percentage of patients with psychiatric illnesses who consulted the doctor peaked in 1972, whereas the percentage of the control group consulting him remained relatively stable over the whole period. The excess over the control group declined more slowly for the group with a new prescription than for the group without a new prescription. As it seemed to take about four years for this excess to halve for the group with a new prescription and about one year for the group without a new prescription, the patients who had psychiatric disorders in 1972, particularly those receiving new prescriptions for psychotropic drugs, had an excess in consultations for physical illness over many years.

\section{Discussion}

Over two decades in a general practice in the United Kingdom the number of consultations for physical illnesses did not decline as a result of the diagnosis of psychiatric disorders and the prescription of psychotropic drugs for these patients; indeed, the opposite was the case. Thus no offset effect occurred and, in contrast with reports of the effects of specialist mental health care in the United States, mental health care from a general practitioner in the United Kingdom did not seem to reduce demand for general medical care.

There is little reason to suspect that the general practitioner's mental health care practices were different from those of general practitioners in the United Kingdom. His rate of detection of psychiatric cases was similar to that found in a large random sample of British general practitioners. ${ }^{12}$ Also, in 1972 the conspicuous psychiatric morbidity of adults in this sample was $19 \cdot 5 \%$, which compares with the pooled prevalence for psychiatric morbidity of $14 \%$ reported in a large study in Greater London. ${ }^{8}$ The percentage of adults in this study who received a new prescription for psychotropic drugs $(7 \cdot 8 \%)$ may be compared with the rates of prescription estimated by Parish $(12 \cdot 6 \%),{ }^{14}$ and by Skegg et al (19.3\%). ${ }^{15}$ Nor is there reason to suspect bias in the general practitioner's general health care; when the percentages of men and women aged 25 and over consulting the general practitioner at least once in 1972 for any reason were compared with those obtained from the second national morbidity survey in general practice for November 1971 to November $1972^{16}$ the relevant percentages were similar $(59.6 \%$ in this study $v 58 \cdot 6 \%$ in the survey for men and $71 \cdot 1 \% v$ $67 \cdot 3 \%$ for women).

Most of the patients with psychiatric disorders ( $85 \%$ 
(81/96) of those with a new prescription and $82 \%$ (117/143) of those without a new prescription) had one or more consultations in the index year for conditions diagnosed by the general practitioner as depressive neurosis. In addition 58\% (56/96) and 36\% (51/143) respectively had one or more consultations for conditions diagnosed by the general practitioner as anxiety neurosis, a result in keeping with the generally accepted view that most psychiatric disorders seen in general practice are of depression with or without associated anxiety. It may, however, be added that general practitioners and psychiatrists find such disorders difficult to define, categorise, or diagnose reliably. ${ }^{17} 18$

Patients diagnosed as having psychiatric disorders were subdivided into those thought to require, or not require, some new psychotropic drug treatment within a chosen year to allow comparison with previous work. ${ }^{3}$ The groups were defined as receiving, or not receiving, a drug not prescribed in the past six months. When the data were coded hypnotic drugs were excluded from the category of psychotropic drugs, and it is possible that patients in either group may have received repeat prescriptions for psychotropic drugs, but we cannot investigate these issues further because of the nature of the original coding. In any case the general conclusion is not affected as both groups had consultations for physical disorders in excess of the control group; it is possible, however, that the excess in such consultations in both groups was attenuated by treatment with psychotropic drugs.

Our results complement those from studies that established a positive association between psychiatric and physical disorder in general practice. In our study a higher percentage of patients diagnosed by the general practitioner as having psychiatric disorders, particularly patients receiving new prescriptions for psychotropic drugs, had consulted the general practitioner for physical illnesses and this excess continued over many years. The excess seems to take about four years to halve for the group receiving new prescriptions for psychotropic drugs, but only about one year for the group not receiving new prescriptions to begin with. Why this is so remains unclear, though consideration of how these groupings are made may throw some light on the findings.

To begin with, patients consult the general practitioner about the vicissitudes of life, variously expressed in physical, psychological, and social terms. His allocation of diagnoses and treatment creates the morbidity labels, such as those specified in this study. The groups thus identified become indicators of the general practitioner's awareness that the patients are "troubled." Those not diagnosed as having psychiatric disorders consult least often and are least troubled. Those diagnosed as having psychiatric disorders are recognised as being more troubled; their trouble is identified by the general practitioner as anxiety and depressive neurosis. The groups consist of a mix of patients with acute and chronic psychiatric disorders. Chronic psychiatric disorder is positively associated with other forms of chronic ill health ${ }^{8}$; patients in both groups have been troubled in this way for around 10 years (figure). One group is perceived to be more troubled than the other: the patients needing new prescriptions of psychotropic drugs have more acute psychiatric disorders or have chronic disorders in exacerbation, both of which are considered serious enough by the general practitioner to require some new treatment. Hence patients given new prescriptions, being more troubled, have a higher rate of consultations over a longer period than patients not given new prescriptions.
It is worth noting that "static" patients in general practice such as those in this sample may receive less medical attention than more itinerant patients. For example, Baker studied the pattern of contact with doctors of static patients in a north east London dormitory suburb between 1951 and $1965 .{ }^{20}$ These patients required less medical attention (to the extent of one third of an item of service a year for men and three quarters of an item for women), but even so requests for medical care rose by $75 \%$ over the period. He concluded that the increase was spread fairly evenly over the range of ages between the sexes and was not distorted by large demands by only a few patients. Unfortunately, it is not possible to tell whether the patients attending frequently in 1951 were those attending in 1965 . Our data do not throw light on this issue of frequent attendance, only whether patients attended at all for physical illnesses.

It has been suggested that much of the offset effect may be due to the way that the physical symptoms accompanying mental disorders are perceived by patients and managed by mental health specialists. ${ }^{21}$ Thus mental health care could substitute for general health care inasmuch as it might relieve the distress caused by physical symptoms. Through such mental health care patients could learn that their perceived physical symptoms were less appropriate for medical care than they formerly believed. Our results from general practice, however, do not confirm this view.

We thank Eileen Andrew for coding of the data. The work was carried out under the direction of Professor Michael Shepherd at the general practice research unit, Institute of Psychiatry, London, with the support of the Department of Health and Social Security.

1 Follette W, Cummings NA. Psychiatric services and medical utilisation in a prepaid health plan setting. Med Care 1967;5:25-35.

Kessler LG, Steinwachs DM, Hankin JR. Episodes of psychiatric care and medical utilization. Med Care 1982;20:1209-10.

3 Hankin JR, Kessler LG, Goldberg ID, Steinwachs DM, Starfield BH. A longitudinal study of offset in the use of non-psychiatric services following longitudinal study of offset in the use of non-psychiatric ser.

4 Schlesinger HJ, Mumford E, Glass GV, Patrick C, Sharfstein S. Mental health treatment and medical care utlization in a free-for-service system: outpatient

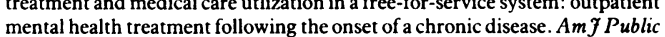
mental health treatme $1983 ; 73: 422-9$.

5 Budman SH, Demby AB, Feldstein ML. A controlled study of the impact of mental health treatment on medical care utilization. Med Care 1984;22: 216-22.

6 Mumford E, Schlesinger HJ, Glass GV, Patrick C, Cuerdon T. A new look at evidence about reduced cost of medical utilization following mental health treatment. Am f Psychiatry 1984;141:1145-58.

7 Borusy JF, Olendzki MC, Kessler L, et al. The "offset effect" of mental health treatment on ambulatory medical care utilization and charges. Arch Gen Psychiatry 1985;42:573-80.

8 Shepherd M, Cooper B, Brown AC, Kalton G. Psychiatric illness in general practice. Oxford: Oxford University Press, 1966.

9 Wilkinson G. Mental health practices in primary care settings. An annotated bibliography 1977-1985. London: Tavistock, 1985.

10 Brown AC, Fry J. The Cornell Medical Index Health Questionnaire in the identification of neurotic patients in general practice. $\mathcal{J}$ Psychosom Res 1962;6:185-90.

11 Cooper B. A study of 100 chronic psychiatric patients identified in general practice. Brf Psychiatry 1965;111:595-605.

12 Skuse $D$, Williams $P$. Screening for psychiatric disorder in general practice. Psychol Med 1984;14:365-77.

13 Dunn G, Skuse D. The natural history of depression in general practice: stochastic models. Psychol Med 1981;11:755-64.

14 Parish PA. The prescribing of psychotropic drugs in general practice. $f(R \mathrm{Coll}$ Gen Pract 1971;21(Suppl 4):1-77.

15 Skegg DCG, Doll R, Perry J. The use of medicines in general practice. BrMed f 1977; i:1561-3.

16 Royal College of General Practitioners. Morbidity statistics from general practice 1971-1972. Second national study. London: HMSO, 1979. (Studies on 1971-1972. Second national study. Lon

17 Jenkins R, Smeeton N, Marinker M, Shepherd M. A study of the classification of mental ill-health in general practice. Psychol Med 1985;15:403-9.

18 Wilkinson $\mathrm{G}$. A comparison of psychiatric decision-making by trainee general practitioners and trainee psychiatrists using a simulated consultation model. practitioners and trainee psychic
Psychol Med 1988;18:167-77.

19 Eastwood MR, Trevelyan MH. Relationship between physical and psychiatric distwood MR, Trevelyan MH. Relation
disychol Med 1972;2:363-72.

20 Baker CD. A fifteen-year pattern of patient-doctor contact among "static" general-practice patients. BrMed f 1966;ii:106-7.

21 Mechanic D. Does psychiatric care reduce the demand for medical care services? Med Care 1983;21:1126-7.

(Accepted 12 May 1988) 